UAB-TH-521

March 2002

\title{
A Stückelberg formalism for the gravitino from warped extra dimensions
}

\author{
Tony Gherghetta ${ }^{a}$ and Alex Pomarol ${ }^{b}$ \\ ${ }^{a}$ School of Physics 8 Astronomy, University of Minnesota, \\ Minneapolis, MN 55455, USA \\ ${ }^{b}$ IFAE, Universitat Autònoma de Barcelona, \\ E-08193 Barcelona, Spain
}

\begin{abstract}
We consider supersymmetric theories with a warped extra dimension where supersymmetry is broken by boundary conditions that preserve an $R$-symmetry. It is shown that this supersymmetry breaking mechanism naturally invokes the Stückelberg formalism for the gravitino in order to give a four-dimensional theory with a smooth massless limit.
\end{abstract}


1. The idea of the unification of forces makes us believe that the Standard Model (SM) is replaced, at high energies, by a more fundamental theory with larger symmetries. Two interesting possibilities are grand unified theories and supersymmetric theories. Of course, the extra symmetries of the fundamental theory must be broken, by some mechanism, down to the SM symmetry group. Extra dimensions allow for new possibilities to break the symmetries of the more fundamental theory down to the Standard Model. The idea is that these symmetries could be realized in the higher dimensional theory but not in four dimensions. It is during the process of compactification that the symmetries are broken. Therefore it is interesting to analyze the breaking of symmetries by boundary conditions and to study its consistency.

In this Letter we want to study the fate of gravitinos in five-dimensional (5d) warped spaces when supersymmetry is broken by boundary conditions. It has been shown [1] that in these theories an $R$-symmetry (which is a chiral symmetry) can be preserved and, as a consequence, there are two light gravitinos, $\Psi_{\mu L}$ and $\Psi_{\mu R}\left(\Psi_{\mu L, R}=\frac{1}{2}\left(1 \pm \gamma_{5}\right) \Psi_{\mu}\right)$, that combine to form a Dirac field. If the effective scale on the boundary where supersymmetry is broken is the $\mathrm{TeV}$ scale, then the gravitino mass is of order $10^{-3} \mathrm{eV}$ (instead of order the $\mathrm{TeV}$ scale as would be the case for a flat $5 \mathrm{~d}$ space). One of the gravitinos, $\Psi_{\mu L}$, is weakly coupled with $1 / M_{P}$-suppressed couplings, and is similar to the usual four-dimensional ( $4 \mathrm{~d}$ ) supersymmetric theories with supersymmetry broken at the TeV scale. Therefore its presence is not unusual. However, the presence of another light gravitino (due to the $R$-symmetry), $\Psi_{\mu R}$, is different from ordinary scenarios. Furthermore, it has a coupling suppressed only by the supersymmetry breaking scale $1 / \mathrm{TeV}$ (which will be explained later why this is the case). This raises the following apparent inconsistency. Since this gravitino is coupled to a nonconserved current $J_{\mu}$ (because supersymmetry is broken) its contribution to an amplitude $\mathcal{M}$ is

$$
\mathcal{M}=\bar{J}^{\mu} P_{\mu \nu} J^{\nu}
$$

where $P_{\mu \nu}$ is the propagator of a massive gravitino

$$
P_{\mu \nu}=\frac{1}{p^{2}+m^{2}}\left[\left(\eta_{\mu \nu}+\frac{p_{\mu} p_{\nu}}{m^{2}}\right)(-i \not p-m)-\frac{1}{3}\left(\gamma_{\mu}+i \frac{p_{\mu}}{m}\right)(i \not p-m)\left(\gamma_{\nu}+i \frac{p_{\nu}}{m}\right)\right]
$$

We are assuming a Minkowski metric $\eta_{\mu \nu}=(-1,1,1,1)$. Notice that this amplitude diverges in the limit $m \rightarrow 0$. In particular, for the coupling of the gravitino $\Psi_{\mu, R}$ to the photon and photino via the fermionic current

$$
\bar{J}^{\mu}=\frac{g}{2 \sqrt{2} M} \overline{\widetilde{\lambda}} \gamma^{\mu} \gamma^{\nu \rho} F_{\nu \rho}\left(1-\gamma_{5}\right)
$$


we can see, using dimensional analysis, that the amplitude $\mathcal{M}$ grows with the energy $E$ as

$$
\mathcal{M} \sim \frac{E^{4}}{m^{2} M^{2}} .
$$

Clearly, this amplitude will overwhelm unitarity bounds at energies $E \gtrsim \sqrt{M m}$. For $M \sim M_{P}$ this consistently occurs at energies $E \gtrsim \mathrm{TeV}$, and there are no problems in the effective theory. However, for $M \sim \mathrm{TeV}$, this implies that the theory is not consistent at energies $E \gtrsim 0.1 \mathrm{MeV}$, which is much below the effective scale which is at the TeV. We will see how this apparent inconsistency is dealt with in the compactification of a higher-dimensional warped theory.

Before we consider supersymmetry and warped geometry it is instructive to consider a simpler example where similar features occur. In fact the above situation is similar to the case of a massive spin-1 particle coupled to a nonconserved current. The Lagrangian is given by

$$
\mathcal{L}=-\frac{1}{4} F_{\mu \nu} F^{\mu \nu}-\frac{1}{2} m^{2} A_{\mu} A^{\mu}+A_{\mu} J^{\mu},
$$

where $J_{\mu}$ is the vector current containing the matter fermions. The propagator of a massive spin-1 particle is given by

$$
\frac{1}{p^{2}+m^{2}}\left[\eta_{\mu \nu}+\frac{p_{\mu} p_{\nu}}{m^{2}}\right]
$$

and becomes singular in the limit $m \rightarrow 0$. Therefore the gauge boson contribution to the four-fermion amplitude grows with energy like $g^{2} E^{2} / m^{2}$, where $g$ is the gauge coupling, and will saturate the unitarity bounds at energies $E \sim m / g$. In this case the procedure for achieving a smooth limit $m \rightarrow 0$ is known. If we add a scalar field $\Phi$ with negative kinetic term

$$
\mathcal{L}_{\Phi}=\frac{1}{2} \partial_{\mu} \Phi \partial^{\mu} \Phi+\frac{1}{m} \Phi \partial_{\mu} J^{\mu}
$$

then the four-fermion amplitude will receive an extra contribution mediated by $\Phi$ that cancels the singular term $\frac{p_{\mu} p_{\nu}}{m^{2}}$ coming from the massive gauge boson. This can also be explicitly seen by performing the shift $A_{\mu} \rightarrow A_{\mu}+\partial_{\mu} \Phi / m$ in the Lagrangian (5), and together with (7) leads to the combined Lagrangian

$$
\mathcal{L}=-\frac{1}{4} F_{\mu \nu} F^{\mu \nu}-\frac{1}{2} m^{2} A_{\mu} A^{\mu}+A_{\mu} J^{\mu}+m \Phi \partial_{\mu} A^{\mu}
$$

In this form the field $\Phi$ appears as a Lagrange multiplier which can be integrated out. This is achieved by introducing a term $-\frac{\mu^{2}}{2} \Phi^{2}$ in the Lagrangian, performing 
a Gaussian integration, and taking the limit $\mu \rightarrow 0$. This finally leads to the Lagrangian

$$
\mathcal{L}=-\frac{1}{4} F_{\mu \nu} F^{\mu \nu}-\frac{1}{2} m^{2} A_{\mu} A^{\mu}+A_{\mu} J^{\mu}+\frac{1}{2 \alpha}(\partial \cdot A)^{2},
$$

with $\alpha=\mu^{2} / m^{2} \rightarrow 0$. This is the Lagrangian for a massive vector field and in the massless limit $(m=0)$ the last term corresponds to the Landau gauge-fixing term. The propagator now becomes

$$
\frac{1}{p^{2}+m^{2}}\left[\eta_{\mu \nu}-\frac{p_{\mu} p_{\nu}}{p^{2}}\right]
$$

and there is no singular term in the limit $m \rightarrow 0$. This is known as the Stückelberg formalism [2] . The field $\Phi$ has the same couplings as a Nambu-Goldstone boson of the global U(1) but its kinetic term has an opposite sign. Therefore it acts like a "ghost" (with negative energy and negative norm). In the massless limit $(m \rightarrow 0)$ and gauge-symmetric limit $\left(\partial_{\mu} J^{\mu}=0\right), \Phi$ cancels the extra degree of freedom of the massive spin-1 boson leaving only the two degrees of freedom of the massless gauge boson

This particular way of achieving a smooth $m \rightarrow 0$ limit using the Stückelberg formalism is, as we shall discuss below, the same way that warped higher dimensional theories recover a smooth $m \rightarrow 0$ limit in the gravitino contribution to amplitudes.

2. Let us consider a $5 \mathrm{~d}$ supersymmetric theory compactified on the orbifold $S^{1} / Z_{2}$, where the two boundaries are located at $y^{*}=0$ and $y^{*}=\pi R$. Fields living in the $5 \mathrm{~d}$ bulk must have definite boundary conditions. For fermions, we can assume that

$$
\psi_{L, R}(0)= \pm \psi_{L, R}(0), \quad \psi_{L, R}(\pi R)= \pm \psi_{L, R}(\pi R)
$$

If their supersymmetric bosonic partners have the same boundary condition, the theory will remain supersymmetric after compactification.

Let us consider the effect of changing the boundary conditions at $y^{*}=\pi R$ from those at $y^{*}=0$ but only for the fermions. This means that the $5 \mathrm{~d}$ fermions of the theory satisfy

$$
\psi_{L, R}(0)= \pm \psi_{L, R}(0), \quad \psi_{L, R}(\pi R)=\mp \psi_{L, R}(\pi R) .
$$

\footnotetext{
${ }^{1}$ Notice that this is different from a non-linear realization of the gauge symmetry, which in the $\mathrm{U}(1)$ case, is sometimes also called the Stückelberg formalism.
} 
These boundary conditions break supersymmetry but preserve a $\mathrm{U}(1)_{R}$ symmetry. This mechanism is usually called Scherk-Schwarz supersymmetry breaking [3]. In a flat 5 d space the action for the gravitino $\Psi_{M},(M=\mu, 5)$, is given by

$$
S_{5}=-\int d^{5} x \sqrt{-g}\left[i \bar{\Psi}_{M} \gamma^{M N P} D_{N} \Psi_{P}+\left[i \bar{J}^{M} \Psi_{M}+\text { h.c. }\right]\right]
$$

and has an $N=2$ supersymmetry. If we impose the boundary conditions of Eq. (12) then the $N=2$ supersymmetry is spontaneously broken to $N=0$. In other words, the $N=2$ supersymmetry is still realized but only nonlinearly. After compactification all the gravitinos are massive. In particular, the lowest modes consist of two gravitinos, $\Psi_{\mu L}$ and $\Psi_{\mu R}$, with a mass $m=1 /(2 R)$. The components $\Psi_{5 L}$ and $\Psi_{5 R}$ play the role of Goldstinos $\mathbb{1}$, and can be eliminated by choosing the unitary gauge

$$
\Psi_{\mu L} \rightarrow \Psi_{\mu L}-\frac{1}{m} \partial_{\mu} \Psi_{5 L}, \quad \Psi_{\mu R} \rightarrow \Psi_{\mu R}+\frac{1}{m} \partial_{\mu} \Psi_{5 R} .
$$

The spectrum of this theory preserves an $R$-symmetry.

For warped spaces the situation is different. Let us consider the metric

$$
d s^{2}=a^{2}(y) \eta_{\mu \nu} d x^{\mu} d x^{\nu}+d y^{2}
$$

Although we believe that our results are valid for a general warp factor $a(y)$, for concreteness, we will focus on the Randall-Sundrum (RS) scenario [5], where $a(y)=e^{-k|y|}$, and $1 / k$ is the curvature radius which is assumed to be of order the fundamental scale. The $5 \mathrm{~d}$ gravitino action is given by [6]

$$
S_{5}=-\int d^{5} x \sqrt{-g}\left[i \bar{\Psi}_{M} \gamma^{M N P} D_{N} \Psi_{P}+i \frac{3}{2} \frac{a^{\prime}}{a} \bar{\Psi}_{M} \gamma^{M N} \Psi_{N}+\left(i \bar{J}^{M} \Psi_{M}+\text { h.c. }\right)\right] .
$$

Let us impose the boundary conditions Eq. (12) on the gravitino wavefunction. This leads to the zero-mode gravitino wavefunctions

$$
f_{L}(y) \simeq \sqrt{2 \pi k R} e^{-\frac{1}{2} k|y|}\left(e^{4 k(|y|-\pi R)}-1\right),
$$

and

$$
f_{R}(y) \simeq 2 \sqrt{\pi k R} e^{\frac{k}{2}(|y|-4 \pi R)}\left(e^{2 k|y|}-1\right) .
$$

Consequently, we see that the gravitino zero-mode $\Psi_{\mu L}$ is localized on the boundary at $y^{*}=0$ (where the effective scale is $M_{P}$ ), while $\Psi_{\mu R}$ is localized on the boundary at $y^{*}=\pi R$ (where the effective scale is $\mathrm{TeV}$ ). This will lead to a $4 \mathrm{~d}$ theory with the features described in the introductory remarks. 
As in the $5 \mathrm{~d}$ flat space case, we would like to know whether the $4 \mathrm{~d} N=1$ supersymmetry is nonlinearly realized by trying to eliminate the fields $\Psi_{5 L, R}$. If we attempt to go to the unitary gauge, using the equivalent redefinitions for the 5 d warped space (which are given in Eq. (12) of Ref. [1]), we find that $\Psi_{5 L}$ is eliminated but that contrary to the flat case, the field $\Psi_{5 R}$ does not disappear from the theory. Instead, we find that the zero-mode gravitino $4 \mathrm{~d}$ Lagrangian is given by

$$
\begin{aligned}
\mathcal{L}_{4} & =-i \bar{\Psi}_{\mu L} \gamma^{\mu \nu \rho} \partial_{\nu} \Psi_{\rho L}-i \bar{\Psi}_{\mu R} \gamma^{\mu \nu \rho} \partial_{\nu} \Psi_{\rho R} \\
& +\left[i m \bar{\Psi}_{\mu L} \gamma^{\mu \nu} \Psi_{\nu R}+i \bar{J}^{\mu} \Psi_{\mu R}+i \sqrt{\frac{3}{2}} m \bar{\Psi}_{\mu R} \gamma^{\mu} \Psi+\text { h.c. }\right],
\end{aligned}
$$

where $m \simeq \sqrt{8} k e^{-2 k \pi R}$ is the gravitino mass, and $\Psi$ is a linear combination of the Kaluza-Klein fields of $\Psi_{5}$

$$
\Psi(x) \simeq \sqrt{12 \pi k R} e^{-\frac{1}{2} \pi k R} \sum_{n=0}^{\infty} \frac{f_{R}^{(n)}(\pi R)}{m_{n}} \Psi_{5 R}^{(n)}(x) .
$$

Notice that in Eq. (19) we have only considered the current to be coupled to $\Psi_{\mu R}$, and have neglected the couplings to $\Psi_{\mu L}$ since they are suppressed by $1 / M_{P}$. As we discussed earlier, the presence of a light gravitino coupled to the nonconserved current of Eq. (3) leads to a photon-photino scattering amplitude ${ }^{2}$

$$
\mathcal{M}_{3 / 2}=\bar{J}^{\mu} \frac{(-i \not p)}{p^{2}+m^{2}}\left[\eta_{\mu \nu}+\frac{2}{3} \frac{p_{\mu} p_{\nu}}{m^{2}}\right] J^{\nu},
$$

that diverges in the limit $m \rightarrow 0$. However, the interesting feature in Eq. (19) is that the field $\Psi$ appears as a Lagrange multiplier in an analogous way to the scalar field $\Phi$ in Eq. (8). This suggests that the fermion $\Psi$ will play a similar role to the scalar field $\Phi$ in recovering the smooth limit $m \rightarrow 0$. In fact, this is exactly what happens. If we perform the following redefinitions

$$
\begin{aligned}
\Psi_{\mu L} & \rightarrow \Psi_{\mu L}-\frac{1}{\sqrt{6}} \gamma_{\mu} \Psi, \\
\Psi_{\mu R} & \rightarrow \Psi_{\mu R}-\sqrt{\frac{2}{3}} \frac{1}{m} \partial_{\mu} \Psi,
\end{aligned}
$$

then the gravitino and Stückelberg fermion, $\Psi$, decouple from each other in the Lagrangian (19), and leads to the separate Lagrangians

\footnotetext{
${ }^{2}$ Notice that using Eq. (3) we have $\bar{J} \cdot \gamma=\gamma \cdot J=0$.
} 


$$
\begin{aligned}
& \mathcal{L}_{3 / 2}=-i \bar{\Psi}_{\mu L} \gamma^{\mu \nu \rho} \partial_{\nu} \Psi_{\rho L}-i \bar{\Psi}_{\mu R} \gamma^{\mu \nu \rho} \partial_{\nu} \Psi_{\rho R} \\
&+\left[i m \bar{\Psi}_{\mu L} \gamma^{\mu \nu} \Psi_{\nu R}+i \bar{J}^{\mu} \Psi_{\mu R}+\text { h.c. }\right], \\
& \mathcal{L}_{\Psi}=i \bar{\Psi} \gamma^{\mu} \partial_{\mu} \Psi+\left[i \sqrt{\frac{2}{3}} \frac{1}{m} \partial_{\mu} \bar{J}^{\mu} \Psi+\text { h.c. }\right] .
\end{aligned}
$$

The Lagrangian (25) is the analogue of Eq. (7) in the massive vector field example. We now see from Eq. (25) that the fermion $\Psi$ gives a new contribution to the amplitude of Eq. (21), namely

$$
\mathcal{M}_{\Psi}=\bar{J}^{\mu} i \frac{2}{3} \frac{p_{\mu} p_{\nu}}{m^{2}} \frac{\not p}{p^{2}} J^{\nu} .
$$

Thus, combining the two contributions (21) and (26) we see that the divergent piece of Eq. (21) is precisely cancelled leading to the combined total amplitude

$$
\mathcal{M}=\mathcal{M}_{3 / 2}+\mathcal{M}_{\Psi}=\bar{J}^{\mu} \frac{(-i \not p)}{p^{2}+m^{2}}\left[\eta_{\mu \nu}-\frac{2}{3} \frac{p_{\mu} p_{\nu}}{p^{2}}\right] J^{\nu},
$$

which is smooth in the limit $m \rightarrow 0$. Again the similarity with the vector field case is quite clear.

We have seen that the presence of the Stückelberg fermion $\Psi$ guarantees that the amplitudes of the theory remain well behaved at energies above the gravitino mass. Unlike the vector field example where the Stückelberg scalar was introduced by hand, in the supersymmetric example the Stückelberg fermion results from the compactification of the warped geometry. However, in order that the above cancellation takes place it is crucial that the Stückelberg fermion $\Psi$ has a kinetic term of opposite sign compared to the other fermions in the theory -see Eq. (25). Classically this is not a problem because $\Psi$ is a fermion, and its free energy is bounded from below (in fact this is a marked improvement compared to the Stückelberg scalar where classically the energy is unbounded). Furthermore, at the quantum level, in processes where the gravitino and $\Psi$ appear off-shell, this will not present a problem either. Nevertheless, the contribution of $\Psi$, Eq. (26), has a pole at $p^{2}=0$ revealing that $\Psi$ can be produced on-shell with negative probability. This is difficult to interpret in quantum mechanics. The fermion $\Psi$ behaves like a "ghost" of negative norm. Although it is not clear how to make sense of this ghost in production processes, it is interesting to notice that the Lagrangian of Eqs. (24) and (25) maintains its structure at the one-loop quantum level, i.e., ultraviolet divergences can be absorbed by the counterterms of the tree-level couplings. This property is due to the fact that the theory consists of two sectors, namely, the Lagrangian of Eq. (24) that corresponds to 
a spontaneously broken supersymmetric theory in the unitary gauge, and the Lagrangian of Eq. (25) that contains the fermion $\Psi$ which behaves as a Goldstino of a global supersymmetry but with an opposite sign kinetic term. Thus, each sector is protected by its own supersymmetry (at least at the one-loop level). This property makes these theories theoretically interesting [7].

A similar situation to the above but for the case of gravitons instead of the gravitino is present in the model of Ref. [8]. In this theory the graviton is massive and a smooth massless limit is achieved due to the presence of a ghost [9] that turns out to be the radion of the extra dimension [10].

3. We have seen that breaking supersymmetry by boundary conditions in warped geometries naturally invokes the Stückelberg formalism. Let us now discuss why supersymmetry breaking is qualitatively different in compact $5 \mathrm{~d}$ theories with warped geometry compared to that encountered in $5 \mathrm{~d}$ flat spaces. The important thing to note is that in flat space supersymmetry is realized in the bulk of $S^{1} / Z_{2}$, and there is no need for boundary terms. Supersymmetry is always a good symmetry of the bulk independently of the boundary conditions of the fields. If boundary conditions are different between bosons and fermions then supersymmetry is realized nonlinearly from a $4 \mathrm{~d}$ perspective. The $4 \mathrm{~d}$ unitary gauge for the gravitinos corresponds to the 5 d gauge $\Psi_{5}(x, y)=0$.

The situation changes in warped spaces. Supersymmetry in warped spaces requires particular boundary couplings for the fields. For example, in the RS scenario with the boundary conditions (11), supersymmetry requires [6] a cosmological constant term on the two boundaries, $\Lambda_{(0)}$ and $\Lambda_{(\pi R)}$, related to the bulk cosmological constant $\Lambda$ in the following way

$$
\Lambda_{(0)}=-\Lambda_{(\pi R)}=-\Lambda / k
$$

These are precisely the conditions needed for the RS metric [5]. If we now change the boundary conditions of the bulk fermions (but not the boundary couplings) then supersymmetry is no longer realized. There is an explicit breaking on the boundary. Consequently, $\Psi_{5}(x, y)$ cannot be gauged away as in the flat case. This explains the appearance of $\Psi_{5}$ in the spectrum. Let us be more explicit. If the fermions of the theory satisfy the boundary conditions (12), then the relation between the cosmological constants required by supersymmetry is no longer given by Eq. (28) but instead given by

$$
\Lambda_{(0)}=\Lambda_{(\pi R)}=-\Lambda / k
$$

These relations, however, do not lead to the RS metric and in fact, do not even lead to a static configuration [11]. If we insist on obtaining the RS metric we 
must add an explicit supersymmetry breaking term of the form

$$
\int d^{5} x 2 \frac{\Lambda}{k} \delta(y-\pi R)
$$

This term only breaks one of the two bulk supersymmetries. Due to Eq. (12), the supersymmetry parametrized by $\eta_{L}$ vanishes on the boundary at $y^{*}=\pi R$, and is insensitive to terms on that boundary. Only the supersymmetry parametrized by $\eta_{R}$ is broken explicitly, and it is for this reason that $\Psi_{5 R}$ cannot be eliminated from the spectrum.

The appearance of the Stückelberg fermion seems to occur in theories with (1) a localized gravitino at $y^{*}=0,(2)$ supersymmetry broken at the opposite boundary at $y^{*}=\pi R$, and (3) $R$-symmetry invariance. The first two conditions are necessary if one wants to explain the hierarchy between $M_{P}$ and the $\mathrm{TeV}$ scale by a warp factor. Therefore the only possibility to avoid the presence of the Stückelberg fermion is to relax the third condition and break the underlying $R$-symmetry. For example, this will be the case for any supersymmetry breaking mechanism that generates a Majorana mass for the gravitinos and gauginos on the $y^{*}=\pi R$ boundary. This will make all the modes of the gravitino $\Psi_{\mu R}$ heavy (with masses of order $\mathrm{TeV}$ ), and the problem of unitarity discussed above will not arise (see Ref. [1, 12] for an example). In this modified scenario the effective warped model at low energies contains only one light gravitino, and we recover an explicit realization of the usual scenarios considered in phenomenological studies of superlight gravitinos [13].

Let us finally comment on similar possibilities involving gauge symmetries. Either in flat or warped space the gauge symmetry can be realized in the bulk without the need for boundary terms. For example, in an Abelian gauge theory, the Lagrangian is given by

$$
\int d^{4} x d y \sqrt{-g} \frac{1}{4} F_{M N} F^{M N}
$$

which is invariant under the transformation $A_{M} \rightarrow A_{M}+\partial_{M} \theta$, irrespective of whether the space is flat or warped. Therefore the breaking of the gauge symmetry by boundary conditions is always nonlinearly realized with $A_{5}$ playing the role of the Nambu-Goldstone boson.

Acknowledgments: T.G. thanks the CERN Theory Division for hospitality where part of this work was done. A.P. was partially supported by the CICYT Research Project AEN99-0766 and DURSI Research Project 2001-SGR-00188. 


\section{References}

[1] T. Gherghetta and A. Pomarol, Nucl. Phys. B 602, 3 (2001) arXiv:hep-ph/0012378.

[2] See for example, C. Itzykson and J. B. Zuber, "Quantum Field Theory," New York, USA: McGraw-Hill (1980) 705pp. (International Series In Pure and Applied Physics).

[3] J. Scherk and J. H. Schwarz, Phys. Lett. B 82, 60 (1979); Nucl. Phys. B 153, 61 (1979).

[4] I. Antoniadis and M. Quiros, Phys. Lett. B 416, 327 (1998) arXiv:hep-th/9707208; I. Antoniadis, S. Dimopoulos and G. R. Dvali, Nucl. Phys. B 516, 70 (1998) arXiv:hep-ph/9710204.

[5] L. Randall and R. Sundrum, Phys. Rev. Lett. 83, 3370 (1999) arXiv:hep-ph/9905221.

[6] T. Gherghetta and A. Pomarol, Nucl. Phys. B 586, 141 (2000) arXiv:hep-ph/0003129; R. Altendorfer, J. Bagger and D. Nemeschansky, Phys. Rev. D 63, 125025 (2001) [arXiv:hep-th/0003117]; N. AlonsoAlberca, P. Meessen and T. Ortin, Phys. Lett. B 482, 400 (2000) arXiv:hep-th/0003248; A. Falkowski, Z. Lalak and S. Pokorski, Phys. Lett. B 491, 172 (2000) arXiv:hep-th/0004093; E. Bergshoeff, R. Kallosh and A. Van Proeyen, JHEP 0010, 033 (2000) arXiv:hep-th/0007044.

[7] N. Boulanger, M. Henneaux and P. van Nieuwenhuizen, JHEP 0201, 035 (2002) arXiv:hep-th/0201023.

[8] R. Gregory, V. A. Rubakov and S. M. Sibiryakov, Phys. Rev. Lett. 84, 5928 (2000) arXiv:hep-th/0002072.

[9] G. R. Dvali, G. Gabadadze and M. Porrati, Phys. Lett. B 484, 129 (2000) arXiv:hep-th/0003054.

[10] L. Pilo, R. Rattazzi and A. Zaffaroni, JHEP 0007, 056 (2000) arXiv:hep-th/0004028.

[11] P. Brax, A. Falkowski and Z. Lalak, Phys. Lett. B 521, 105 (2001) arXiv:hep-th/0107257.

[12] D. Marti and A. Pomarol, Phys. Rev. D 64, 105025 (2001) arXiv:hep-th/0106256. 
[13] T. Gherghetta, Phys. Lett. B 423, 311 (1998) arXiv:hep-ph/9712343; A. Brignole, F. Feruglio and F. Zwirner, Nucl. Phys. B 516, 13 (1998) [Erratum-ibid. B 555, 653 (1998)] |arXiv:hep-ph/9711516]; S. Gopalakrishna and J. Wells, Phys. Lett. B 518, 123 (2001) arXiv:hep-ph/0108006. 